\title{
Abstracts of the
}

\section{3rd Canadian Neuro-Oncology Meeting}

\section{Summary}

The 3rd Canadian Neuro-Oncology Meeting was held in London, Ontario, on June 9-10, 1988. The meeting was attended by over 100 neurosurgeons, neurologists, radiation oncologists, medical oncologists, neuropathologists, neuroradiologists, scientists, oncology nurses, residents and graduate students. Fifty-five papers were presented covering a wide range of topics in basic and clinical neuro-oncology. Paediatric brain tumours, brain tumour biology, angiogenesis and brain edema, NMR spectroscopy, brain tumour imaging, prognostic factors in malignant glioma, new therapeutic approaches to malignant glioma, malignant glioma chemotherapy, treatment toxicity and management of metastasis were the major areas of focus at the meeting.

In attendance were four outstanding guest speakers. Dr. Peter Berger, Professor of Pathology at Duke University Medical Centre, discussed the "Anatomy, Classification and Dedifferentiation of Astrocytic Neoplasms". Dr. Bengt Westermark, Professor of Tumour Biology at the University of Uppsala in Uppsala, Sweden, reviewed the current understanding of "Oncogenes and Growth Factors in Malignant Glioma". Dr. James Goldie, Professor of Medicine and Chief of Medical Oncology at the Cancer Control Agency of British Columbia, called upon extensive experience in solid tumour chemotherapy and developed "A Rational Approach to Malignant Glioma Chemotherapy". Dr. Clifford Schold, Associate Professor of Medicine (Neurology) at Duke University Medical Centre, emphasized the importance of methodology in his lecture entitled "Design and Conduct of Clinical Trials in Neuro-Oncology".

The majority of participants were Canadian but there was a substantial American contribution to the 1988 meeting. Forty percent of papers were delivered by residents, fellows or graduate students. This reflects a substantial and growing interest in neurooncology in Canada. The 4th Canadian Neuro-Oncology Meeting will be held in Winnipeg in 1990 under the direction of Dr. D. Fewer. 
1.

\section{Medulloblastoma: Astrocytic Differentiation Potential in Short} Term Cell Culture

B.L. MARIA, P.A. STECK, W.K.A. YUNG, A. MILICI and F.F. BECKER (Houston, U.S.A.)

Medulloblastoma is one of the most common and malignant childhood neoplasms. Cellular heterogeneity and the presence of astrocytic (glial fibrillary acidic protein; GFAP) and/or neuronal markers (neuronspecific enolase; NSE) of differentiation in medulloblastoma tumor specimens is associated with a poor survival rate. There is considerable controversy as to this tumor's cell(s) of origin and the exact nature of its differentiation potential in vivo. The purpose of the present study was to examine the effects of differentiation-inducers on medulloblastoma in short term cell culture. A portion of tumor specimen containing GFAP focally ( $<5 \%$ of cells) from a 10-month-old child with recurrent posterior fossa medulloblastoma was grown in monolayer culture (RB2). Cellular morphology, western immunoblotting and/or immunohistochemistry for GFAP and NSE were examined in RB2 before and after treatment with differentiation-inducers.

RB2 in culture consisted of small polygonal cells (90\%), large flat polyhedral cells (5-10\%) and polygonal cells with cytoplasmic processes $(<5 \%)$. In untreated RB2, 30\% of cells expressed GFAP and staining for NSE was negative. Retinoic acid treatment produced flattening of cells and a decrease in GFAP. Dibutyryl cyclic AMP induced the appearance of fine cytoplasmic processes containing GFAP in $90 \%$ of cells within $9 \mathrm{~h}$. Neither agent induced the appearance of NSE.

These results suggest that RB2 cells in monolayer culture have a potential for astrocytic differentiation. Hence, immunohistochemistry for GFAP in medulloblastoma surgical specimens may underestimate the number of cells from an astrocytic lineage. Furthermore, short term cell culture may be useful in examining the relationship(s) between medulloblastoma, other primitive neuro-ectodermal tumors and normal cerebellar histogenesis.

2.

Continuous Infusion Vincristine and Adriamycin (CIVA) for Recurrent Childhood Brain Tumors

R.W. WALKER, M.T. JENNINGS and M. MCELWAIN (New York, U.S.A.)

Childhood brain tumors have a poor prognosis in general, but at recurrence are invariably fatal. Although Vincristine (VCR) has been well-studied in central nervous system neoplasms, there is less experience with Adriamycin (ADR). We have tried a new chemotherapeutic approach administering these two agents as a continuous rather than rapid infusion. Fourteen patients, aged 8-35, with recurrent brain tumors have been treated. The diagnoses were medulloblastoma 9 , germinoma 1, embryonal carcinoma 1, primitive neuroectodermal tumor 1, ependynoma 1 , and malignant meningioma 1 . Of the medulloblastoma patients, 3 had systemic recurrences (pelvis 1 and bone 2); the others were confined to the central nervous system. All 14 patients had previously been irradiated, and 11 had failed prior chemotherapy. VCR $\left(3 \mathrm{mg} / \mathrm{m}^{2}\right)$ and ADR $\left(45 \mathrm{mg} / \mathrm{m}^{2}\right)$ were infused over 72 hours through a central venous line, using disposable pumps changed daily. Treatment courses were given once a month. The number of courses given ranged from 1-10. One patient died of pneumonia immediately after the first course of CIVA and was considered inevaluable for response. In the 13 evaluable patients response was documented by $\mathrm{CT}$, bone scan, or myelogram. There was 1 complete response of $34+$ months duration and 5 partial responses of $2+, 3+, 6(2)$, and 7 months. Five patients had stable disease for $3,3+, 7,8+$, and 8 months. Two patients had progressive disease. Toxicity was tolerable and primarily hematologic. Transfusions were necessary in those patients heavily pretreated with chemotherapy. There was no cardiotoxicity. There was 1 instance of drug extravasation due to a dislodged catheter, and 4 episodes of presumed Broviac sepsis. CIVA has demonstrated an impressive response rate in this preliminary study. Enduring remissions have been achieved even in those patients failing prior chemotherapy regimens.

\section{3.}

\section{Brachytherapy for Recurrent Posterior Fossa Tumours in Children}

\section{N.J. LAPERRIERE, H.J. HOFFMAN, R.P. HUMPHREYS and P.M.K.} LEUNG (Toronto, Ontario)

We report our early experience with brachytherapy in 5 children with recurrent posterior fossa tumours. All children had undergone pre or post-operative external radiation therapy at presentation. Four of the children had received prior chemotherapy as either part of initial management or for recurrence. Histologically, 3 cases were medulloblastomas and 2 were ependymomas. All cases had local recurrence only with no evidence of craniospinal seeding. All patients underwent a repeat craniotomy with resection of as much tumour as was possible. At that same operation, low activity iodine-125 seeds were permanently implanted into residual non-resectable tumour. The prescribed dose was 100 Gray at $0.5 \mathrm{~cm}$ from the plane of the array of seeds. To date, there have been 2 post-operative deaths: one due to aspiration pneumonia in a patient who had a large amount of residual neoplasm in her brain stem, and one death due to meningitis. The other 3 patients are at home and well at 2 years, 7 months, and 1 month since their implant. We conclude that this experimental therapy may be of potential benefit for selected children with recurrent posterior fossa tumours, and that the risk of post-operative complications may be higher than at presentation in these children because of their extensive prior therapy.

\section{4.}

\section{Pineal Region Tumours in Children}

\section{JENKIN (Toronto, Ontario)}

From 1959-1986 51 consecutive children, less than 17 years old, underwent primary treatment at HSC for a tumour in the pineal region. The histological diagnoses were germinoma 21 patients, endodermal sinus tumour 2 patients, choriocarcinoma 2 patients, pineoblastoma 7 patients, teratoma 4 patients, astrocytoma 4 patients and no tissue diagnosis 11 patients.

In $21 / 40(52 \%)$ patients the tissue diagnoses was germinoma. It is assumed that a similar proportion of unbiopsied patients had germinoma. All but 1 biopsied patients were diagnosed after 1971 .

After ventriculo-peritoneal shunting (VPS) unbiopsied patients were irradiated. $9 / 11(82 \%)$ survived. All patients received local irradiation (LRT).

20/21 patients with germinoma were irradiated following VPS, exploration and biopsy. The unirradiated patient was deeply comatose post-operatively and died without further treatment. 19/20 irradiated patients are currently alive. One patient died suddenly 2 months postoperatively of uncertain cause. No tumour was found at autopsy. Three patients relapsed at spinal cord sites, 2 after local irradiation and one after 2 days of elective spinal irradiation. $0 / 20$ irradiated patients died due to progressive germinoma.

Current treatment options will be discussed.

\section{5.}

Analysis of Glioma Cellular Heterogeneity Using Human Monoclonal Antibodies

\section{DAN, J. GUY, S. MAYER and G. PRICE (Montreal, Quebec)}

Three human monoclonal antibodies $(\mathrm{HmAb})$, designated BT27/1A2, BT27/2A3, and BT32/A6 were prepared by fusing B-lym- 
phocytes from patients with malignant gliomas to HGPRT deficient human myeloma-like cell line, TM-H2-SP2, and screening for surface reactivity to the human glioma cell line, SK-MG-1, using an ELISA assay. Flow cytometric studies of SK-MG-1 revealed that the latter consists of 2 populations of cells, referred to as 'small' cells and 'large' cells, and that all $3 \mathrm{HmAb}$ selectively labelled only the 'small' cells. Ten random and independently selected clones of SK-MG-1 each contained the dual populations of the parental line, with preservation of 'small' cell labelling by the $3 \mathrm{HmAbs}$. Using the flow cytometer to sort cells according to size and fluorescence, 10 'small' cell and 10 'large' cell-derived clones (S101-10 and L101-10, respectively) were generated and compared with the parental line. Again, each of the clones contained dual cell populations, but with some important phenotypic and genotypic differences. The 'small' and 'large' cell populations of the $\$ 100$ clones, and the 'large' cells of the L100 clones appeared to be identical to SK-MG-I, whereas the 'small' cells of the L100 clones were physically smaller than those of the parental line. In addition, average DNA content, as assessed by propidium iodide staining, was higher for both 'small' and 'large' cells of the L100 clones, when compared to SK-MG-1. Statistical analysis of metaphase chromosomes from $3 \mathrm{~S} 100$ and L100 clones revealed wide fluctuations and higher average chromosome number for the L100 clones ( $<<0.05$ ), but essentially no difference between the S100 clones and SK-MG-1. Sorted 'small' and 'large' cells of SK-MG-1 grew with equal efficiency when plated out in adherent cultures, however, selective growth of 'small' cells was favoured at low fetal calf serum concentrations. We conclude that the human glioma line SK-MG-1 is a heterogeneous cell line, and that our $3 \mathrm{HmAbs}$ bind preferentially to a population of 'small' cells in both the parental line and its respective clones. The exact relationship between 'small' and 'large' cells is uncertain, since both appear to give rise to each other. 'Large' cell-derived clones appear to differ phenotypically and genotypically from SK-MG-1, whereas 'small' cell-derived clones resemble the parental line more closely. At a functional level, the minority 'small' cells may represent the principal stemline for SKMG-1, and the 'large' cells, sidelines which are maintained in a state of suppression by the former.

6.

Further Investigation of the Transport of Sarcnu, a Sarcosinamide Analog of Chloroethylnitrosourea in Human Glioma Cells

\section{SKALSKI, L. PANASCI and W. FEINDEL (Montreal, Quebec)}

SarCNU is a chlorethylnitrosourea with a sarcosinimide (methyl glycinamide) carrier. SarCNU was previously shown to be less toxic in mice than BCNU and significantly more active than BCNU against a human glioma cell line implanted intracerebrally in nude mice. Comparative studies of BCNU vs SarCNU demonstrated that SarCNU is less myelotoxic than BCNU in the CFU-C assay and more active than BCNU against primary human glioma cells in the Human Tumor Cloning assay. The presence of an amino acid amide moiety on SarCNU suggested a possible existence of a carrier-mediated transport for this compound in contrast to BCNU which enters cells by passive diffusion. The mode of entry of SarCNU into the human glioma cells, SK-MG-1 was examined indirectly by using cytotoxicity as an indicator of transport. The cytotoxicity of SarCNU (and BCNU) was evaluated in media plus or minus excess amounts of either amino acids or sarcosinamide in order to determine whether SarCNU would be less toxic in the presence of these potential competitors for transport. The cytotoxicity of SarCNU was not affected by the amino acids; however it was significantly reduced in the presence of excess sarcosinamide. This finding prompted an examination of the uptake of ${ }^{3} \mathrm{H}$-sarcosinamide by the SK-MG-1 cells. Preliminary results show that the accumulation of sarcosinamide in these cells is slightly above unity at saturation. The uptake of this amino acid amide was also found to be temperature dependent and sodium independent. The transport of the radiolabelled sarcosinamide is inhibited by excess cold sarcosinamide and SarCNU but not by BCNU, glycine or methylglycine. These findings are indicative of facilitated diffusion as the mechanism by which sarcosinamide enters SK-MG-1 cells. Studies aimed at defining the kinetic parameters and possible natural substrates of this transport system are presently in progress.

\section{7.}

Quantification of Protein Synthesis in Experimental Brain Tumor: Rate Constants for Valine Incorporation into Protein

\section{S. MITSUKA, M. DIKSIC, Y.L. YAMAMOTO and W. FEINDEL (Montreal, Quebec)}

Autoradiographic measurement of protein synthesis requires an accurate knowledge of kinetic constants in the specific pathological condition.

Quantitative autoradiography was used to estimate the rate constants for valine incorporation into protein in AA ascites tumor implanted into rat brain. Seven days after implantation, tumors grew up to $5 \mathrm{~mm}$ in diameter with minimum necrosis. Ten $\mathrm{UCi}$ of $\mathrm{L}-1-14 \mathrm{C}$-valine was administered and the animals decapitated at intervals to determine the time course of tissue and plasma activity. The rate constants were calculated by a non-linear, least square fitting procedure, with the equation derived from three compartment and four rate constant models for incorporation of carboxyl labeled valine into protein.

Since the distribution of the tracer in the tumor is heterogeneous, tissue activity was measured from two different regions, namely the whole tumor and the region of highest accumulation of the tracer. Calculated rate constants in the tumor were as follows:

\begin{tabular}{|c|c|c|c|c|}
\hline Rate Constants & & Normal Gray & Tumor Mean & Tumor Peak \\
\hline$K_{1}$ & $(\mathrm{ml} / \mathrm{g} / \mathrm{min})$ & $0.038 \pm 0.005$ & $0.294 \pm 0.059$ & $0.534 \pm 0.0 \% 0$ \\
\hline$k_{2}+k_{3}$ & $(/ \min )$ & $0.116 \pm 0.020$ & $0.115 \pm 0.096$ & $0.141 \pm 0.062$ \\
\hline$k_{4}$ & $(\mathrm{~min})$ & $0.033 \pm 0.005$ & $0.058 \pm 0.050$ & $0.094 \pm 0.036$ \\
\hline Precursor half-life & $(\min )$ & 4.7 & $5.41 \pm 2.70$ & $3.49 \pm 1.19$ \\
\hline Distribution volume & $(\mathrm{m} l / \mathrm{g})$ & 0.255 & $2.09 \pm 0.80$ & $2.59 \pm 0.73$ \\
\hline $\begin{array}{l}\text { Net clearance } \\
\text { of valine }\end{array}$ & $(/ \mathrm{g} / \mathrm{min})$ & 0.0084 & $0.098 \pm 0.014$ & $0.215 \pm 0.016$ \\
\hline
\end{tabular}

The values of $K_{1}, k_{4}$, distribution volume and net clearance of valine in both mean and peak accumulated region and $k_{2}+k_{3}$ in the peak region in the tumor were significantly higher than in the normal brain. The rate of valine incorporation into protein in this tumor was 12 - 26 times higher than that of brain tissue.

The same method was applied to ipsi- and contralateral cerebral cortex in the tumor-bearing rat brain. These results indicate that protein synthesis in the brain tissue, adjacent to the tumor, is similar, whereas glucose utilization rates in these structures have been reported to be reduced. Furthermore, the ratio of the metabolic rate between tumor and brain is much higher in the protein method than the deoxyglucose method.

This research was supported by the NIH (NS 22230).

\section{8.}

Primary Brain Tumours with Enclosed Neurons and Cortical Dysplasia Associated with Focal Seizures

SHI TING HUI, S. GAYTAN-GARCIA, J. GIRVIN, W. BLUME and J.C.E. KAUFMANN (London, Ontario)

During a span of 13 years and 10 months (February 1974 to December 1987) 256 patients underwent unilateral lobectomy or corticectomy at the University Hospital to control drug resistant seizures. Neuropathological examination showed primary brain tumours in 85 cases (33\%); gliomas has a notable preponderance over lesions other than gliomas (79:6). Among these gliomas, astrocytomas were the most common with 38 cases $(45 \%)$; oligodendrogliomas 16 cases $(19 \%)$; mixed gliomas 10 cases $(12 \%)$; gangliogliomas 8 cases $(9.2 \%)$; and glioblastoma multiforme 7 cases $(8.2 \%)$. The remaining lesions were 1 meningioma $(1.1 \%) ; 1$ pineal germinoma $(1.1 \%) ; 1$ sarcoma of the leptomeninges $(1.1 \%) ; 1$ glial cyst $(1.1 \%) ; 1$ epidermoid cyst $(1.1 \%)$ and 1 
porencephalic cyst $(1.1 \%)$. In general the great majority of the tumours were of small size and in an early stage of growth; as a result the changes in the background had not yet been obliterated by tumour growth.

Apart from the morphological changes directly relating to the brain tumours, there were abnormalities indicating disturbance of neuronal migration and maturation. Coexistence of gliomas and cortical dysplasia may provide some evidence for a relationship between dysgenetic abnormalities and neoplasm and could be the morphological substrate in cases of brain tumours associated with seizures. In addition the neuronal density in the epileptogenic tumours was measured compared with the site and type matched controls; there are statistically significant differences between the two groups.

9.

Angiogenesis and Vasculogenesis in the Murine C6 Astrocytoma Spheroid Implantation Model

R.F. DEL MAESTRO, C.L. FARRELL, J. MEGYESI and P. COSTELLO (London, Ontario)

The continued growth of both experimental and human glial tumours is dependent on the generation of new capillary vessels: angiogenesis. Angiogenesis may be considered a "cascade" of dependent morphological and biochemical events which are modulated by different microenvironments. Conceptually in the angiogenic cascade, initiation events are those changes which occur in specific endothelial cells and their basement membranes which begin the process of antiogenesis and occur before endothelial cell proliferation occurs. Endothelial cell proliferation occurs subsequently which lengthen the loops of new microvessels and finally termination events can also occur in some tissues.

This angiogenic cascade of events is being examined using the murine C6 astrocytoma spheroid implantation model. Tumour angiogenesis occurs early after implantation of an avascular spheroid of $\mathrm{C} 6$ cells and, by 5 days post-implantation, new microvessels of high permeability are well-established. Although angiogenesis continues to occur, a second phenomena, vasculogenesis, in which tumour cells induce changes in previously normal blood vessels, becomes a prominent feature of tumour growth. Vasculogenesis is associated with the outgrowth of tumour cells along previously established blood vessels altering both their biochemical and morphological appearance and in part determining both the invasiveness and subsequent growth of the tumour. These changes are felt to be related to the release of specific factors from tumour cells which act at a distance from the major tumour mass. The inter-relationship between specific angiogenic factors and the phenomenon of vasculogenesis is unknown. However, it is clear that continued growth of murine C6 astrocytoma spheroid tumours after implantation involves a complex interplay between angiogenesis and vasculogenic events.

Supported by the Brain Research Fund Foundation.

10.

The Accumulation of Polymorphonuclear Leukocytes in Experimental and Human Glial Tumours

C.D. LORDO, W. MCDONALD, A. STEELE and R.F. DEL MAESTRO (London, Ontario)

The mechanism(s) responsible for the development of edema associated with the growth of intracerebral tumours are not completely understood. One mechanism which may be involved in the microvascular permeability alteration seen is the presence of an inflammatory reaction to tumour growth. To assess the role of the inflammatory response in tumour growth, the accumulation of polymorphonuclear leukocytes (PMNs) into tumour and peritumour tissue was studied using the murine $\mathrm{C} 6$ astrocytoma spheroid implantation model and human tumours. Polymorphonuclear leukocytes were quantified using a spec- trophotometric assay for the PMN specific enzyme myeloperoxidase. Myeloperoxidase (MPO) is a hemoprotein which catalyzes the peroxidation of a wide variety of substrates, comprises up to 5\% of the dry weight of PMNs and is almost exclusively found in PMNs. A leukocyte common antibody that stains for PMNs in fixed tissue was also used to histologically assess the PMN infiltration.

The accumulation of PMNs as measured by MPO activity was significantly increased in tumour tissue as compared to peritumoural and contralateral hemisphere in the C6 astrocytoma model. Monoclonal antibody staining supported these results demonstrating PMNs both attached to tumour microvessel endothelium and in the tumour tissue itself. The MPO assay also demonstrated substantial PMN localization with specimens taken at operation from patients with glioblastoma multiforme. These results confirm the presence of significant numbers of PMNs in both an experimental and human glial tumours and suggest that an inflammatory response may be a component of tumour growth.

Supported by Upjohn and Brain Research Fund Foundation.

11.

\section{The Functional Microcirculation in a Glioma Model}

C.L. FARRELL, R.F. DEL MAESTRO, C.R. FARRELL and P.A. STEWART (London and Toronto, Ontario)

The functional characteristics of the microvasculature of tumors in the brain are different from those in normal brain tissue. In order to investigate the blood flow in the microvasculature of a glioma, we examined individual vessel perfusion in tumors of rats implanted with C6 spheroids. Current techniques using in vivo microscopy do not permit quantitative analysis of patterns of vascular perfusion in intracranial tumors. Thus we used the following three techniques to examine the microcirculatory function in this C6 glioma. The brains of one group of rats implanted with spheroids were fixed and stained using the alkaline phosphatase technique which, in this glioma model, appears to stain all the vessels in the tumor tissue. A second group of implanted rats were perfused continuously with Aquablak ink for 3 minutes; this appears to fill all the perfusable vessels in the tumor and normal brain tissue. A third group of implanted rats were injected with Horseradish peroxidase (HRP) and decapitated after 15 seconds. HRP mixes rapidly with blood when injected intravenously and marks only those vessels that are perfused during the single passage of the tracer through the brain in the 15 second time interval. We used image analysis to count vessel density in 89 sections from a total of 13 tumors in these 3 groups. All tumors used were less than $7 \mathrm{~mm}^{2}$ in maximum cross sectional area and had no necrosis. During the single passage of blood through the cerebral microvasculature, only $40 \%$ of the tumor vessels are perfused and we defined this population as active vascular volume. Only $80 \%$ of the total number of vessels were perfusable with Aquablak and we defined this as the perfusable vascular volume. The vessel densities in these two groups were significantly different from that of the alkaline phosphatase stained preparations. This heterogeneous perfusion of individual microvessels in viable tumor tissue may account for the low blood flow observed clinically and experimentally in intracranial tumors. The microvessels in most brain tumors are very permeable and so the flux of a substance into a tumor is probably critically dependent on flow in the active vascular volume. This information may be important for the design of better treatments for tumor-associated edema and chemotherapeutic drug delivery.

Supported by the National Cancer Institute of Canada and the Brain Research Fund Foundation.

12.

Electron Microscopic Analysis of Cerebral Microvascular Endothelium In Vitro

\section{P. COSTELLO and R.F. DEL MAESTRO (London, Ontario)}

Endothelium, the site of the blood-brain barrier (BBB), 1 has been 
shown to be morphologically different in neoplastic cerebral microvessels in comparison with endothelium of non-neoplastic microvessels in the brain. ${ }^{2}$ The BBB in tumours shows altered thickness, increased vesicle density in glial tumours and decreased junctional indices. Human endothelium derived from neoplastic and non-neoplastic tissue samples have been isolated and cultured in our laboratory. Cell lines from glial tumours and from 'normal' brain tissue samples were designated as endothelium if they stained positive for Factor VIII antigen and isolectin B4. The morphological appearance was a cobblestoned pattern. These cell lines were then plated at an early passage (1-3) onto Lux Thermanox coverslips and allowed to grow to confluency. Endothelium were also grown in co-culture with glial cells, normal and neoplastic. The BBB cells were then processed for examination under the electron microscope. Sections of samples from 10 patients were analyzed for their ultrastructural properties. The hypothesis that the tumour derived endothelial cells maintain altered characteristics in vitro was tested using junctional index, vesicle density and overall cell fragility as criteria. The endothelial cells from both tissue sources were treated identically throughout their in vitro life and therefore any discrepancies would be due to the difference in the tissue of origin. The endothelium from tumours have a lower junctional index and are more fragile than endothelium from normal human brain. There was no statistical difference in the vesicle density but some tumour derived endothelium contained abnormal vesicular bodies. These endothelial cell properties may reflect the comprised BBB of tumours seen in vivo.

IBradbury MWB. The structure and function of the blood-brain barrier. Fed Proc 1984; 43: 186-190.

2Stewart PA, et al. Quantitative study of microvessel ultrastructure in human peritumoural brain tissue. J Neurosurg 1986; 67: 697-705.

13.

\section{Correlation of Tumor Vascular Ultrastructure with Peritumoral} Edema in Human Meningiomas

P.A. STEWART, C.L. FARRELL and R.F. DEL MAESTRO (Toronto and London, Ontario)

The blood-brain barrier (BBB) is a protective mechanism that regulates the passage of blood-borne molecules into the brain. Under normal conditions, the selective permeability of this interface is maintained, in part, by the almost total absence of large pores in the vessel walls. Vascular pores in the form of fenestrations, vesicular channels and junctional channels, do, however, occur in vessels within brain tumors, and their non-specific leakiness results in the formation of vasogenic edema. It has been suggested that the type and density of the pores are different in different tumor types, and that this variation accounts, at lease in part, for the known variability in tumor-associated edema and in the vulnerability of different tumors to blood-borne antineoplastic drugs.

Meningiomas in the human are associated with significant amounts of cerebral edema. The aim of the present study is to determine which type(s) of pores occur in human meningiomas, measure their density in tumor tissue, and determine which of them correlates with the extent of edema.

Samples of meningioma were collected during surgery for removal of the tumor, fixed within a few seconds of removal from their blood supply and processed for routine electron microscopy. Vessel profiles sectioned approximately transversely were randomly selected and photographed using a Hitachi 7000 electron microscope. Negatives of the vessel profiles were projected onto the surface of a digitizing tablet and the fenestrae, junctional clefts and vesicular density were measured as previously detailed (Microvasc Res 1987; 33: 270-282). To evaluate tumor-associated edema, contrast-enhanced CT scans were obtained for each patient and the volumes of the tumor, the low-density edematous area and the total brain volume containing both tumor and edema were measured using computer-assisted image analysis.

We found that meningioma vessels had numerous fenestrations $(87$ per $\mathrm{mm}$ luminal surface length, compared with 0 in normal brain and 0 in glioblastoma multiforme vessels), and significantly enlarged junc- tional cleft indices (59\% compared with $26 \%$ in normal brain tissue) but only very slightly increased vesicular densities (7.2/um ${ }^{2}$ compared with $3.2 / \mathrm{um}^{2}$ in normal brain vessels and $60-80 / \mathrm{um}^{2}$ in highly permeable non-brain vessels). The volume of edematous tissue ranged from $75 \%$ of the tumor volume to almost twice the tumor volume. Patients with the highest volume of edma also had the highest density of fenestrae. We conclude that vasogenic edema associated with meningiomas is primarily due to interendothelial junctional abnormalities and fenestrations, and that vesicular-based permeability is not important in this tumor type.

This work was supported by the National Cancer Institute of Canada and by the Brain Research Fund. RFD is the recipient of an Ontario Ministry of Health Career Scientist Award.

\section{4.}

Positron Emission Tomography, Computed Tomography and Angiographic Correlations in Untreated Cerebral Astrocytomas

\section{J.-G. VILLEMURE, V.G. WAGLE, J.L. TYLER, J.F. EMRICH and D. MELANSON (Montreal, Quebec)}

Cerebral astrocytomas are heterogeneous tumors with varying pathological features. The tumor imaging modalities available today each contributes different information about the tumor and its relationship to the surrounding brain. While CT provides primarily structural information and angiography in addition shows vascularity, PET has the unique capability of providing, non-invasively, information on the in-vivo metabolism of tumors.

We studied 13 patients with proven gliomas with infused CT, angiography, and PET prior to treatment. Correlations between these studies and clinical, operative and pathological findings were established. A solid or cystic appearance on CT, avascularity or vascularity on angiography, duration of clinical symptoms and tumor grade were compared to cerebral blood flow (CBF), cerebral blood volume (CBV), and glucose metabolism (LCMRGI). The following trends emerged.

1) CBV was consistently high in 12 of 13 tumors whether they were cystic or solid, avascular or hypervascular.

2) CBF was reduced in cystic tumors and variable in solid tumors

3) Glucose metabolism was variable and showed no relation to pathological grade, CT, or angiographic findings.

4) All high grade (III, IV) gliomas had high CBV and variable CBF and LCMRGI.

5) CBF and LCMRGI were low in patients with new onset of symptoms, high in those with a 3-week to 2-month duration of symptoms and variable in those with symptoms greater than 2 months.

We conclude that PET in cerebral astrocytomas provides unique information on metabolic parameters that cannot be predicted from CT or angiography. The findings on PET confirm the heterogenous nature of these tumors. With magnetic resonance imaging and spectroscopy, further information on the structural and metabolic aspects of these tumors will be obtained. This information will ultimately help in designing treatment protocols and following the effects of therapy.

15.

\section{Non-enhancing Malignant Gliomas}

\section{D.R. MACDONALD, J.G. CAIRNCROSS and J.J. GILBERT (London, Ontario)}

The CT scan appearance of cerebral gliomas often predicts the tumor histology: malignant gliomas (MG) typically have prominan contrast enhancement, well-differentiated "low grade" gliomas (LGG) usually are low density masses with minimal or no enhancement. In the past 5 years we have seen 11 patients ( 7 women, 4 men; ages 24-64 years; median, 30 ) with MG in whom the history and CT scan suggested LGG (4.5\% of all patients with MG seen during this time). Symptoms preceded diagnosis by 1 month to 7 years (median, 10 months) and included seizures in 8 patients. Tumors were temporal in 3 
patients, temporal-parietal in 2, frontal in 2 and bifrontal, parietal, occipital and thalamic in 1 patient each. On CT scan all tumors were low density masses involving white matter; none had contrast enhancement, 3 had calcification. Tumor pathology was anaplastic astrocytoma (AA) in 10 patients and glioblastoma multiforme (GBM) in 1. All received radiotherapy and 10 chemotherapy; 1 patient with AA died 5 months after diagnosis (autopsy showed GBM); 10 remain well $4+$ to $36+$ months after diagnosis (median, $16+$ months). Some patients with MG have non-enhancing tumors on CT scan. As the CT scan may not accurately predict tumor histology, all patients with suspected gliomas should have a biopsy to establish diagnosis and guide treatment.

16.

\section{Patterns of Failure Following Treatment for Glioblastoma Multiforme and Anaplastic Astrocytoma}

\section{K.E. WALLNER, J.H. GALICICH, G. KROL, E. ARBIT and M.G.} MALKIN (New York, U.S.A.)

Recurrence patterns of glioblastoma multiforme (GBM) and anaplastic astrocytoma (AA) were studied with CT scanning in 34 patients treated at Memorial Sloan-Kettering Cancer Center from 1983 to 1987 . There were 18 males and 16 females. Median age at initial diagnosis was 54 years, with a range of 34-76 years. Twenty-six patients had GBM and eight had AA. Thirty-two patients had unifocal tumors and two had multifocal tumors. All patients had received postoperative radiation therapy. Whole brain irradiation of 40-60 Gy was given in most cases; only one patient received less than 40 Gy to the whole brain. Eighteen patients had re-operation at the time of recurrence with histologic proof of recurrent tumor; 16 patients had only radiographic evidence of recurrence. Eighty-one percent (26/32) of unifocal tumors recurred within $2.0 \mathrm{~cm}$ of the initial tumor margin, defined as the enhancing edge of the CT scan tumor image. Sixty-one percent $(19 / 32)$ of tumors recurred within $1.0 \mathrm{~cm}$ of the initial tumor margin. No tumor recurred more than $3.5 \mathrm{~cm}$ from the initial tumor margin. There was no difference in failure pattern between patients with GBM versus those with AA. Generally, larger tumors were not more likely to recur further from the initial tumor margin than were smaller tumors. Extensive edema was not associated with a propensity for tumors to recur further from the initial tumor margin. Totally resected tumors tended to recur closer to the initial tumor margin than did subtotally resected tumors $(p>0.1)$. No unifocal tumor recurred as a multifocal tumor. The data presented support the rationale for partial brain irradiation in the postoperative treatment of high-grade astrocytomas, and may help to determine the most appropriate tumor volume for interstitial irradiation.

17.

Comparison of Microcomputer-Based Planimetry with Geometric Models to Quantitate Response of Primary Anaplastic Brain Tumors

R.E. ALBRIGHT, JR. and E.K. FRAM (Durham / San Francisco, U.S.A.)

The total and enhancing tumor volumes from 97 patients (388 CT scans) with primary anaplastic brain tumors were quantitated using microcomputer-based planimetry and three geometric models: (1) an ellipse using the maximum orthogonal diameters, (2) a sphere using the maximum cross-sectional diameter, and (3) an ellipse based on the maximum planimetry cross-sectional area (PMAX-E). The enhancing tumor volume was calculated by subtracting the intra-tumor low density (core) volume from the total tumor volume. Patients with multicentric or non-contiguous tumors were excluded from the study. The patient population included 61 males, 36 females, mean age of 52.4 years (range 15.1 to 81.4 y.o.), 69 glioblastoma multiforme, 22 anaplastic astrocytomas, 3 anaplastic oligodendrogliomas, 1 gliosarcoma and 2 non-biopsied lesions. All the tumors were supratentorial except for one pontine lesion. The mean interscan interval was 77 days (range 3 to 652 days).

The changes in tumor volume were quantitated as a direct percent change (\% $\mathrm{CHG}$ ) and as an exponential response rate (RR) relative to the interscan time (units of day-1). Comparison of the geometric models to planimetry was made using linear regression with determination of the correlation coefficient and the $95 \%$ confidence intervals.

Analysis was made of six groups: the \% CHG of the entire group and those samples with \% CHG under $200 \%$ and $100 \%$ and the RR of the entire group and those samples with RR under 5.0 day $^{-1}$ and 2.0 day-I. In four of the six groups, the PMAX-E geometric model had the best $95 \%$ confidence interval and the highest coefficient of correlation compared with the ellipse and spherical models. The best $95 \%$ confidence interval was in the group of samples having a total tumor \% CHG under $100 \%$ or a RR under 2.0 day $^{-1}$ with sample error of $\pm 45.2 \%$ and \pm 0.6 day $^{-1}$, respectively. The spherical model showed the greatest error of measurement in all groups. The PMAX-E method quantitated an inverse tumor change to planimetry in 13-20\% of cases, e.g. change indicating tumor increase while planimetry indicated tumor decrease.

The findings indicate that, when compared to planimetry, geometric models have significant inaccuracies in quantitating changes in tumor volume defined by $\mathrm{CT}$. The changes seen in a tumor using CT do not follow a given geometry that would allow a simple method for quantitation. Planimetry is more accurate at quantitating both the direct volume and the serial changes of anaplastic brain tumors using $\mathrm{CT}$.

18.

Cerebral Astrocytomas Without Contrast Enhancement on CT or Focal Mass Effect on CT and MRI

L.R. ROGERS, M.L. ESTES and M.A. WEINSTEIN (Cleveland, U.S.A.)

Diffuse astrocytoma, including gliomatosis cerebri, is an uncommon form of cerebral astrocytoma. The clinical diagnosis of neoplasm is often difficult because of multifocal or diffuse cerebral symptoms. We report three patients with diffuse cerebral astrocytoma in whom the difficulty in diagnosis was compounded because the computed tomography (CT) and magnetic resonance images (MRI) were not suggestive of tumor. CT scans in all revealed low attenuation coefficient in cerebral hemispheric white matter suggestive of vasogenic edema. There was no enhancement following contrast injection in any patient, including double-dose contrast in two. MRI T2-weighted images in two patients revealed increased signal intensity diffusely throughout hemispheric white matter. Two patients had undergone radiation for astrocytoma 15 and 56 months prior and the CT and MRI changes were misinterpreted as radiation effects. The correct diagnosis was established at autopsy (2) or tumor resection (1). Biopsy from an additional patient with similar CT and MRI findings revealed only gliosis and nine months later he developed a multifocal anaplastic astrocytoma. This report underscores the difficulty in the diagnosis of brain neoplasm in these patients because of lack of contrast enhancement on CT and lack of focal tumor or mass effect on CT and MRI.

19.

Intraoperative Measurement of Light Penetration Through Brain During Photodynamic Therapy

\section{P.J. MULLER and B.C. WILSON (Toronto, Ontario)}

The degree of tumor cell killing which occurs during the course of photodynamic therapy (PDT) is dependent on the concentration of the photosensitizer in the tumor cells, the light energy absorbed by the cells, and, the inherent cell sensitivity to the photodynamic effect. The degree of tumor cell light energy absorption is in part dependent on the 
transmission of light through the tissue. Light penetration depth is defined as the distance in $\mathrm{mm}$ that results in a reduction to $37 \%$ in light flux or intensity.

In 8 patients with malignant primary brain tumours, whom we treated with intraoperative cavitary PDT, we determined the penetration depth of light in brain tissue in vivo by reading the detected light flux from a fiber passed radially into the brain towards the centre of the irradiation volume. The optical fiber consisted of a single $400 \mu \mathrm{m}$, cleaved fiber fixed in a 17-gauge biopsy needle coupled to a photometer.

A total of twenty data sets were acquired in vivo. In three patients we measured light flux in tumour, in two patients brain and in 3 patients the measurements were in mixed brain and tumour tissue. We identified considerable variation in the penetration depth from patient to patient and from place to place in the same patient. The light penetration depth ranged from 0.8 to 4.9 . The mean PD $( \pm S D)$ for tumour, tumour-brain and normal brain was $2.9 \pm 1.5,2.4 \pm 1.2$ and $1.5 \pm 0.43$, respectively.

The penetration depth was greater in tumour than in normal brain. We attribute this significant difference to the differences in the absorption and scattering properties of brain and tumour.

20.

\section{Intracarotid Combination Chemotherapy for Recurrent Brain Metastases}

\section{D.J. STEWART, M. RIDING, Z. GRAHOVAC, H. HUGENHOLTZ} and L. EAPEN (Ottawa, Ontario)

Two patients with brain metastases from adenocarcinoma of the lung recurrent after surgical resection and cranial irradiation (both patients) plus systemic chemotherapy (one patient) were treated with intracarotid mitomycin-C, cisplatin, and VM-26 combined with intravenous cisplatin, VM-26, and vincristine. Intracarotid mitomycin-C was scheduled to be administered every 6 weeks, with intracarotid cisplatin and VM-26 every 3 weeks. Cisplatin was given IV for one day and VM-26 was given IV for 2 days after each intracarotid infusion of cisplatin and VM-26 ( \pm mitomycin-C). Vincristine was given IV on the same day as each intracarotid infusion. Intravenous mannitol and oral glycerol were given before and during each day of chemotherapy in an attempt to preferentially augment blood flow (and hence drug delivery) to intracerebral tumor. One patient received only a single intracarotid infusion of mitomycin-C, cisplatin, and VM-26. A second infusion was not given because she was found to have asymptomatic narrowing of the carotid artery. All treatment was continued IV. The second patient had all treatment changed to IV administration when she developed ipsilateral transient periorbital edema and long term opthalmoplegia (without much decrease in visual acuity). Both patients had very marked reduction in tumor size after the first intracarotid infusions accompanied by symptomatic improvement. Responses lasted for 6 months in both patients. Further patient accrual on this study is planned.

21.

\section{A Retrospective Analysis of Supratentorial Malignant Glioma in Adults: Prognostic Factors and Survival}

M.J. WINGER, D.R. MACDONALD, P. BANERJEE and J.G. CAIRNCROSS (London, Ontario)

We report a retrospective analysis of prognostic factors and survival for 285 consecutive adults with newly diagnosed, biopsy-proven, supratentorial malignant glioma ( 188 gliobastoma, 76 anaplastic astrocytoma, 11 mixed malignant glioma, 10 anaplastic oliogodendroglioma) treated at the London Regional Cancer Centre from July 1982 through December 1987. Our approach to initial therapy was similar for all patients and included maximum feasible resection, radiotherapy (RT) and adjuvant chemotherapy. The median survival for all patients was 35 weeks. Multivariate analysis demonstrated that age, duration of symptoms, pre-RT performance status, tumor histology, accessibility to resection (ie., tumor location), extent of resection, radiotherapy and prior suspected or proven low grade glioma were significant independent prognostic variables. The prognostic importance of age, duration of symptoms, performance status and tumor histology have been emphasized by others. We report several "new" findings. First, patients with anaplastic oligodendroglioma had the longest median survival (278 weeks). Second, corrected for accessibility and all other variables, patients with gross total resection lived longer than those with partial resection and patients with any degree of resection live longer than those in whom the tumor was only biopsied. Third, patients with malignant glioma in whom there was a prior history of suspected or proven low grade glioma lived significantly longer from the diagnosis of malignant glioma than did patients in whom the malig. nant glioma arose de novo.

22.

Correlation of Astrocytoma Tumor-Associated Antigen Expression In Vitro with Patient Survival

M.T. JENNINGS, D.L. JENNINGS, L.H. ASADOURIAN, H.T. THALER, S. EBRAHIM, J.R. SHAPIRO and L.J. OLD (New York, U.S.A.)

Previous serologic and cytogenetic study of malignant gliomas has emphasized tumor heterogeneity and implied a certain randomness of phenotypic traits. We have studied the expression of 55 cell surface antigens among early passage normal glia (NG) (8 cell lines), low grade astrocytomas (AST) (4) and 42 malignant gliomas (MG) in vitro. Cell line characterization demonstrated that NG and AST had similar antigenic phenotypes but differed biologically as AST were near-diploid in karyotype and anchorage independent. In contrast, MG were cytogenetically abnormal, anchorage independent and formed nu/nu xenografts. Sixty percent of MG expressed markedly divergent antigenic phenotypes. Sixteen cell surface antigens were found expressed among the MG but not NG or AST lines. These were highly restricted, differentiation and lineage specific antigens of endothelial, epithelial, mesenchymal and nonglial neuroectodermal derivation.

Clinical and histopathologic features of this patient population were correlated with the in vitro characterization. Among the 4 low-grade astrocytoma and 37 malignant glioma patients, diagnosis $(p=0.006)$ and age $(p=0.003)$ were the dominant predictors of survival Correlation was found between diagnosis and the expression of antigens $5.1 \mathrm{HII}(\mathrm{p}=0.001)$, A010 $(p=0.001)$, CAT301 $(p=0.008)$ CNT/10 ( $p=0.027)$ and MIII ( $p=0.034)$. Recurrent tumors demonstrated reduced expression of antigens F24 (p < 0.0005), A010 (p= $0.014)$, GD-2 ganglioside $(p=0.017), \mathrm{Vl}(\mathrm{p}=0.018), \mathrm{S} 6(\mathrm{p}=0.03), \mathrm{S} 4$ $(p=0.04)$, HLA-DR $(p=0.04)$, CNT/10 $(p=0.04)$ and EGF-R $(p=0.06)$. "Unadjusted" analysis demonstrated an unfavorable association between patient survival and expression of antigens $5.1 \mathrm{H} 11$ $(\mathrm{p}=0.002), \mathrm{CNT} / 10(\mathrm{p}=0.04)$, and B2.6 $(\mathrm{p}=0.05)$. Multivariate analysis, correcting for diagnosis and age, demonstrated that HLA-DR $(p=$ $0.02)$ and $5.1 \mathrm{H} 11(\mathrm{p}=0.06)$ were associated with a shorter interval of patient survival. Tumor associated antigenic phenotype in vitro may be the first biologic trait to correlate with the natural history of malignant gliomas and suggests the application of such a model for its predictive utility.

\section{3.}

\section{Dynamic Stereotactic Radiosurgery}

E.B. PODGORSAK, A. OLIVIER, M. PLA, J. HAZEL, L. SOUHAMI and C.R. FREEMAN (Montreal, Quebec)

Radiosurgery is a brain irradiation technique in which a very small radiation field $(\sim 1 \mathrm{~cm}$ diameter $)$ is used to deliver a very large radia- 
tion dose ( $5000 \mathrm{cGy})$ in a single fraction. The aim of radiosurgery is to produce necrosis in the target volume by a single high radiation dose without adversely affecting the surrounding brain tissues. It is used in the treatment of small brain tumours, such as pituitary adenomas, and in certain non-neoplastic conditions, such as inoperable arterio-venous malformations. The requirements for successful radiosurgery are very stringent and range from accurate determination of the target volume, to accurate delivery of the dose in the target volume, both spatially and numerically, and to a very sharp dose fall-off in regions outside the target volume. The practical goal thus is to give to the target volume a very high and relatively uniform dose with a high degree of spatial accuracy concurrently with as low as possible a dose to the rest of the brain tissue.

Radiosurgical procedures are performed with multiple focused cobalt beams obtained from dedicated units incorporating about 200 cobalt sources, with proton beams from cyclotrons, or with high energy $\mathrm{x}$-rays from isocentric linacs. The extreme cost of the first two techniques stimulated the development of cheaper, yet as effective, linac based techniques. In order to achieve a high dose fall-off outside the target volume the linac techniques use either multiple coverging beam arcs or a simultaneous and continuous gantry and couch motion during the treatment (dynamic radiosurgery).

The dynamic radiosurgical technique was developed at the McGill University in Montreal. A stereotactic frame is fixed to the patient's skull and used for target localization, treatment set up, and patient immobilization during the treatment. An isocentric linac (Clinac-18) with a remotely controlled couch rotation is used as the source of $10 \mathrm{MV} x$-rays. The gantry of the linac rotates from $30^{\circ}$ to $330^{\circ}$, while simultaneously the couch rotates from $75^{\circ}$ to $-75^{\circ}$. The circular beam converges at the target volume, but because of the simultaneous couch and gantry rotations, the entrance beam never coincides with an exit beam even though the gantry goes through almost a full $360^{\circ}$ rotation. This method then gives a uniform dose distribution in the target volume and an optimal dose all-off outside the target volume. We will discuss the physical aspects of the technique and show that the necessary additions and modifications on an isocentric linac to incorporate the dynamic radiosurgery are relatively simple, making the dynamic radiosurgery an attractive and inexpensive option in comparison to commercially available dedicated radiosurgical techniques.

24.

\section{Stereotactic Selective Fractionated Radiotherapy of Brain Tumours - Methodology}

A. OLIVIER, E.B. PODGORSAK, M. PLA, A. de LOTBINIERE, J. HAZEL and L. SOUHAMI (Montreal, Quebec)

The development of selective stereotactic fractionated radiotherapy (SSFR) at McGill has been a natural extension of our method of radiosurgery. By contrast to radiosurgery, where a single high dose of $\mathrm{x}$-rays is delivered to a small lesion or area of the brain, selective fractionated therapy consists in the delivery of smaller doses (approximately $700 \mathrm{cGy})$ usually to larger volumes $(2-3 \mathrm{~cm}$ in diameter) in a fractionated manner (in 6 fractions over 2 weeks). Common to both approaches is the stereotactic anatomo-pathological correlation and its relationship with the various isodose levels, in view of delivering maximal doses to selective parts of a tumour and minimal doses to normal brain structures.

The stereotactic assessment of tumour morphology, anatomical structures and isodose planning can be done by CT, MRI and DSA.

The interface with the linear accelerator is achieved by transferring the frame isocentre landmarks to the scalp with a triple laser beam alignment system. A halo is used to immobilize the head to the rotating couch by a special clamp, thus maintaining the target at the isocentre.

The patient can then undergo a series of fractionated treatments using the double continuous rotation technique developed for radiosurgery.

Our preliminary experience, based on the management of five intrinsic brain tumours, will be presented.
25.

Photo-Dynamic Therapy of Malignant Primary Brain Tumours

\section{P.J. MULLER and B.C. WILSON (Toronto, Ontario)}

We are updating our experience with intraoperative PDT in 32 patients with malignant supratentorial gliomas; in 19 cases the tumour was recurrent. There were 20 males and 12 females with an age range of $17-73($ mean $=45)$ years. The first 8 patients in this series received HPD (Photofrin I) and the next 24 received DHE (Photofrin II). A photo-illuminating device, of the authors' design, was coupled to an argon dye pump laser in order to deliver light at $630 \mathrm{~nm}$ to a tumour cavity created by radical tumour resection and/or tumour cyst drainage. The total light energy delivered ranged from 440 to 3888 Joules and the light energy density ranged from 8 to $68 \mathrm{~J} / \mathrm{cm}^{2}$. All patients were kept in a low light intensity environment post-operatively.

There were 2 post-operative deaths as the consequence of hematoma accumulation in an extensive tumour resection cavity. In 2 patients neurological function was worse post-operatively and did not recover. Post-operative cerebral edema was pronounced in 8 cases and required emergency craniotomy in 2 patients (the histology from both showed hemorrhagic necrosis of residual tumour). Four patients developed wound infections; 2 of these required surgical treatment. Four patients, 2 of whom were hemiparetic, developed deep vein thrombosis and required anticoagulant therapy. There were no adverse systemic reactions to the administration of either photosensitizer and only 3 skin photo-sensitivity reactions.

Follow up has ranged from 1 to 3 years. In the interval between PDT and death, the deaths per observation year was 1.02 for the whole group and 0.94 when the 2 post-operative deaths are excluded. In the interval between first diagnosis and death, the rate was 0.47 deaths per observation year. The median post-PDT survival time was 29 weeks with $28 \%$ surviving $>1$ year and $6 \%$ surviving $>2$ years. The median survival time from first diagnosis (1st operation) was 67 weeks with $60 \%$ surviving $>1$ year and $22 \%$ surviving $>2$ years.

We have followed these patients with sequential CT scans. In 6 patients the tumour disappeared on CT scan post PDT. Of this group 4 did not receive any post-PDT radiotherapy or chemotherapy and had declining or no steroid requirement. Two of the 6 patients had postPDT radiotherapy. In the 4 patients who did not receive post-operative radiation therapy the delayed disappearance of the tumour was attributed to PDT. Of the 6 cases 5 have had tumour recurrence and 4 have died. In 4 patients the area of original tumour remained stable or decreased; in spite of which tumour developed remote from the site of treatment suggesting that light penetration was inadequate.

Photodynamic therapy of malignant brain tumours using surface or cavitary photo-illumination can be carried out with acceptable risk and appears to be active against some gliomas.

26.

Radiosensitization with Constant Intra-Arterial (IA) Infusion of Bromodeoxyuridine (BUdR) and Focal External Radiation Therapy (RT) in the Treatment of Malignant Astrocytomas

H.S. GREENBERG, W.F. CHANDLER, R.F. DIAZ, W.D. ENSMINGER, L.R. JUNCK, M.A. PAGE, S.S. GEBARSKI, T.W. HOOD, P.L. STETSON, A.S. LICHTER and R. TANKANOW

We treated 23 patients between July 1985 and March 1988 with continuous IA BUdR infusion and focal external RT. BUdR, a halogenated pyrimidine analog, is incorporated into DNA of dividing cells in competition with thymidine and sensitizes these cells to radiation. Neurons and glia have a low mitotic rate, will not incorporate BUdR and will not be sensitized. BUdR is delivered IA because of a regional advantage which we calculated to be between 5 and 16. An 8-week continuous BUdR infusion was delivered for two weeks before and during focal external RT. Twenty-three patients ( 18 Grade IV, 5 Grade III) received BUdR with dosage escalation from 400 to $600 \mathrm{mg} / \mathrm{m}^{2} /$ day 
with a median duration of follow-up of twenty months. The maximum tolerated dose is $400 \mathrm{mg} / \mathrm{m}^{2} /$ day. The Kaplan-Meier estimated median survival of the 23 patients is 23 months (S.E. \pm 3 months). Three patients have had prolonged disease-free responses of 34+, 30 and $27+$ months. There have been no vascular complications. Side effects include ipsilateral forehead dermatitis, blepharitis and conjunctivitis. One patient developed a Stevens-Johnson syndrome requiring BUdR termination. IA BudR with focal RT may improve survival and induce long term remission in patients with malignant gliomas.

27.

\section{Comparative Glioma-Directed In-Vitro Cytotoxicity}

J.P. FARMER, H. RODE, J.G. VILLEMURE and J.P. ANTEL (Montreal, Quebec)

Modern therapeutic modalities offer an expected survival of only $37-40$ weeks to the patient harboring a glioblastoma multiforme. A search for alternative treatment avenues is needed. Motivated by the positive correlation between degree of lymphocytic infiltration of primary brain tumors and patient survival, we have developed a technique for isolating tumor infiltrating lymphocytes (TIL) from fresh glioma tissue using mechanical dissociation followed by Percoll density gradient centrifugation. TIL's isolated by this method can be phenotypically characterized and then expanded either as bulk populations or clones in IL-2 supplemented in-vitro culture. Antologous peripheral blood lymphocytes (PBL) can be concurrently isolated and cultured in order to permit comparisons with the TIL's. We have been able to expand TIL populations from bulk cultures in 10 patients studied to date. TIL's expanded in-vitro demonstrate lytic activity against glioma target cells in a $5 \mathrm{ICr}$ cytotoxicity assay. Determination of the magnitude and specificity of this activity is currently being undertaken using as targets autologous or allogeneic glioma cells as well as non-glial tumors and allogeneic normal cerebral tissue. The approach should determine the nature of glioma specific cytotoxic $T$ cells with potential for future use as tumor-cidal agents.

28.

Malignant Glioma at a Regional Cancer Centre - Implications for the Interpretation of Clinical Trials

\section{M.J. WINGER, D.R. MACDONALD, D.E. MOULIN, S.C. SCHOLD} and J.G. CAIRNCROSS (London, Ontario)

The London Regional Cancer Centre (LRCC) is a university associated treatment facility and the sole radiotherapy (RT) unit for 1.1 million people. Virtually all patients with malignant glioma are evaluated, treated and followed at this centre. From October 1983 through June 1987, 55 adults with malignant glioma entered a phase III chemotherapy protocol in which randomization occurred 8 weeks after RT. This represented $28 \%$ of the 197 new, biopsy-proven, supratentorial malignant gliomas seen here over the same period. One hundred and fortytwo patients $(72 \%)$ were lost to study; poor functional status was responsible in 83 cases $(42 \%)$. The greatest loss $(32 \%)$ occurred prior to RT and was largely related to poor function. Median survival was 60 weeks for study patients and 25 weeks for non-study patients $(p=0.00001)$. Study patients were not representative of the entire group. They were younger, with less disability and had a more favourable histology than non-study patients. Median survival for the entire group was 35 weeks. This analysis from a centre whose experience with malignant glioma is population-based emphasizes three points. First, most patients with malignant glioma at study centres do not participate in randomized clinical trials. Second, clinical trials study a biased subset of patients with malignant glioma by excluding those who do poorly at the outset. Third, survival predictions for patients with malignant glioma based on the results of randomized clinical trials may be overly optimistic.
29.

Treatment of Malignant Gliomas with "Eight-Drugs-in-One-Day" Chemotherapy Administered Before and After Radiotherapy

J. ROZENTAL, I. ROBINS, D. TRUMP, J. FINLAY, R. STEEVES, B. HEALY and J. COHEN (Madison, U.S.A.)

Thirty-one adult patients with malignant gliomas ( 23 with glioblastoma multiforme, 6 with anaplastic astrocytomas, and 2 with brainstem gliomas) were treated with the "eight-drugs-in-one-day" chemotherapy regimen (methylprednisolone $300 \mathrm{mg} / \mathrm{m}^{2}$, vincristine $1.5 \mathrm{mg} / \mathrm{m}^{2}$ (maximum of $2 \mathrm{mg} /$ cycle), CCNU $75 \mathrm{mg} / \mathrm{m}^{2}$, procarbazine $75 \mathrm{mg} / \mathrm{m}^{2}$, hydroxyurea $3000 \mathrm{mg} / \mathrm{m}^{2}$, cisplatin $90 \mathrm{mg} / \mathrm{m}^{2}$, cytosine arabinoside $300 \mathrm{mg} / \mathrm{m}^{2}$, and imidazole carboxamide $150 \mathrm{mg} / \mathrm{m}^{2}$ ). We planned chemotherapy as 2 cycles before, and 8 cycles after, $60 \mathrm{~Gy}$ of involved brain irradiation. A total of 102 cycles of chemotherapy were administered. There was one treatment-related death. The most frequent toxicity was myelosuppression (leucopenia $<1000 / \mathrm{mm}^{3}$ occurred in 9 cycles, $1000-2500 / \mathrm{mm}^{3}$ in 25 thrombocytopenia $<100,000 / \mathrm{mm}^{3}$ occurred in 34 cycles). Fifteen patients developed infections requiring treatment. Five patients suffered ototoxicity. Eleven patients suffered nausea and vomiting. A reversible rise in creatinine was seen in 4 patients. One patient developed a severe motor neuropathy and two developed mild peripheral neuropathies. Three patients developed episodes of atrial fibrillation. One patient, with a pulmonary embolus, developed a new bundle branch block with supraventricular tachycardia. Five patients developed thrombophlebitis and three of these had pulmonary emboli. Two patients suffered strokes in areas anatomically separate from their tumor. Eight patients declined to continue therapy after an average of 3 cycles. Twenty-six patients are evaluable for response; 2 have had a complete and 5 a partial response. The median survival is 47 weeks. The responses and survival observed are comparable to less toxic treatment protocols for adults with malignant gliomas.

30.

Improved Survival in Adults With Malignant Gliomas Treated With Radiation Therapy and Modified "Eight-Drugs-in-One-Day" Chemotherapy

N. KOBRINSKY, J. LUTZ, D. FEWER, W. HALLIDAY, W. GORDON, K. MALAKER, K. JONES, K. VIJAY, M. CHEANG, N. HECHTENTHAL, L. JONASSON, N. JOHNSON and DIANE RUMPEL (Winnipeg, Manitoba)

Seventeen adult patients with high-grade gliomas diagnosed in 1987 were treated with an intensive multi-agent chemotherapy (CTX) regimen following completion of standard radiation therapy (SRX). The chemotherapy regimen was based on a modification of the "8-in-1" regimen, developed in Seattle, for treatment of pediatric patients, and included vincristine, procarbazine, hydroxyurea, CCNU, methylprednisolone, carboplatin, dacarbazine and cytosine arabinoside, administered with mannitol diuresis over six hours, every six weeks for a total of eight courses. The outcome of these patients was compared to historical controls treated in 1985 and 1986 with SRX alone (23 patients), or with hyperfractionated radiation therapy (HRX) (20 patients). Patients in the SRX and HRX groups had similar outcomes (progressionfree survival $17.0 \pm 2.6$ vs $24.0 \pm 3.4$ weeks, $p=.73$; overall survival, $36.0 \pm 3.2$ vs $34.5 \pm 3.3$ weeks, $p=.91$ ), and were, therefore, combined for the purpose of subsequent analysis. The CTX and SRX/HRX groups were comparable for sex, extent of surgical resection, histologic grade and Kamofsky score. The groups differed in mean age (50 v. 60 years, $p=.01$ ) and radiation therapy dose (49.3 vs $54.6 \mathrm{~Gy}, \mathrm{p}=.0002)$. Treatment with CTX prolonged progression-free survival $(32.0 \pm 2.8$ vs $18.5 \pm 2.4$ weeks, $p=.008)$, and overall survival $(43.0 \pm 2.7$ vs $35.0 \pm 2.4$ weeks, $p<.04$ ), compared with SRX or HRX alone. Treatment with CTX also prolonged survival in 8 patients with recurrent tumors, compared with 34 patients who did not receive salvage CTX (30.0+ vs $10.0 \pm 2.1$ weeks, $p=.0005)$. CTX was administered on 
an outpatient basis in $24 / 82(34 \%)$ courses. Progressive hematologic toxicity $(\mathrm{p}<.05)$, requiring dose reduction $(\mathrm{p}=.001)$ was noted with subsequent courses of therapy. Red blood cell transfusions following $3 / 80(3.8 \%)$, and platelet transfusions following 9/80 (11.2\%) courses of therapy, were required. Infections and deaths related to therapy were not observed. Based on the encouraging findings of this pilot study, a prospective randomized trial should be considered.

\section{1.}

\section{Menogaril in the Treatment of Recurrent Astrocytomas}

D.J. STEWART, H. HUGENHOLTZ, V. DASILVA, M. RICHARD, N. RUSSELL and C. BENOIT (Ottawa, Ontario)

Menogaril, a new anthracycline derivative, has been used to treat 21 patients with recurrent astrocytomas. Fourteen patients received IV menogaril 10 to $115 \mathrm{mg} / \mathrm{m}^{2}$ once weekly, while 7 patients received oral menogaril 120 to $350 \mathrm{mg} / \mathrm{m}^{2}$ once weekly. A far higher dose intensity is possible on a once weekly administration schedule than on other treatment schedules. All patients except one had recurred after prior cranial irradiation and 13 had received prior chemotherapy (including 10 with prior intracarotid chemotherapy). Original tumor grade was II in 6 patients, III in 3 patients, and IV in 7 patients and mixed oligodendroglioma/astrocytoma in 5 patients. With IV menogaril, 3 patients $(21 \%)$ have had $>50 \%$ tumor shrinkage, $2(14 \%)$ have had tumor shrinkage but $<50 \%$ and $2(14 \%)$ have had stable disease. One patient whose grade III astrocytoma had recurred after irradiation and progressed on intracarotid chemotherapy achieved marked tumor reduction with menogaril persisting now for 18 months $(10$ months off treatment). With oral menogaril, 2 patients (29\%) have had tumor shrinkage $<50 \%$, and $1(14 \%)$ had stable disease. The drug is generally well tolerated. Granulocytopenia is dose-limiting. Further studies continue.

Supported by Upjohn Pharmaceuticals.

32.

\section{Malignant Oligodendroglioma: A Chemosensitive Tumor}

\section{D.R. MACDONALD and J.G. CAIRNCROSS (London, Ontario)}

Nine consecutive patients with recurrent malignant oligodendroglioma have responded to systemic chemotherapy. All patients (5 men; 4 women; ages 28-60 years; Karnofsky 70-90) had prior surgical resection and radiotherapy; 4 had prior chemotherapy, 2 as adjuvant treatment and 2 for recurrence. Seven patients received a combination of CCNU, procarbazine and vincristine (PCV-3), 1 received carmustine (BCNU) and 1 diaziquone (AZQ). All responded by clinical and $C T$ scan criteria. One patient had a complete response (disappearance of all enhancing tumor on CT scan, off steroids, neurolgically intact) for 78 weeks; 8 patients had partial responses ( $>50 \%$ reduction in tumor size, steroids reduced, neurologically improved) for 34 to $110+$ weeks. Two patients had complete control of systemic metastases. Three patients continue to respond $(36+, 69+, 110+$ weeks); 6 patients have relapsed locally ( $34-78$ weeks). Chemotherapy has been well tolerated. The prognosis for most patients with malignant glioma recurrent following surgical resection and radiotherapy is poor; $25-50 \%$ respond briefly to chemotherapy (ie., published response rate). Malignant oligodendroglioma appears to be a uniquely chemosensitive glial tumor. The likelihood of 9 consecutive responses occurring by chance if the true response rate is only $25-50 \%$ is low ( $p<0.000004-0.002$; exact binomial test). Nitrosourea based chemotherapy, especially PCV-3, appears to be effective chemotherapy for recurrent malignant oligodendroglioma.

33.

Radiation Brain Damage Due to Interstitial Iodine-125 Implants: An Experimental Model
M. BERNSTEIN, M. HENKELMAN, P. STEWART, T. MAROTTA, J. GLEN and L. RESCH (Toronto, Ontario)

Interstitial brachytherapy is a promising therapy for malignant gliomas. This modality of therapy is currently under investigation in Toronto in a phase III randomized study for newly diagnosed patients, and also in a phase II study for patients with recurrent malignant astrocytomas not participating in the phase III study. One of the limiting factors to the efficacy of this therapy is the development of radiation necrosis within the tumour and surrounding brain necessitating subsequent craniotomy. Another problem is that of differentiating recurrent tumour from radiation effect on follow-up imaging studies such as CT and MRI.

To attempt to study the effects of interstitial irradiation on normal brain, we have developed a small laboratory animal model. High activity lodine-125 seeds are laid on the cerebrum of male F-344 rats and the seeds are left in long enough to administer a dose of $8,000 \mathrm{cGy}$ to a $5.5 \mathrm{~mm}$ radius volume. Cohort groups of animals are then studied at the time points of $0,3,6,9$ and 12 months. Study end-points include MRI with and without gadolinium enhancement, blood brain barrier breakdown as assessed by horseradish peroxidase (HRP) staining, light microscopy, and electron microscopy.

Preliminary studies indicate that all the above end-points are sensitive to low dose-rate radiation brain damage due to lodine-125 seeds. HRP staining corresponds approximately to the $20,000 \mathrm{cGy}$ isodose as does the positive signal on gadolinium-enhanced MRI. Routine histology demonstrates the typical features of radiation necrosis with fibrinoid change in the blood vessels. Further out there is a ring of edema. Electron microscopy demonstrates numerous abnormalities of capillaries such as increased diameter, and twisted contour. Also swelling of astroglial end-feet and increased size of the extracellular space are seen. As well the horseradish peroxidase tracer diffuses beyond the area of frank necrosis. Further study will allow correlation of radiation dose, MRI signal, HRP staining and light and electron microscopic changes.

34.

Cerebral Radiation Necrosis: Imaging Patterns at 11-C-LMethionine Positron Emission Tomography

P.C. PHILLIPS, L.A. O'TUAMA, N.D. LAFRANCE, L. STRAUSS, R.F. DANNALS, H. RAVERT, A.A. WILSON, B. CARSON and H.N. WAGNER JR. (Baltimore, U.S.A.)

Radiation-induced brain injury, an important clinical problem in the brain tumor patient, was estimated in one report to have an incidence of $5 \%$. The distinction between radiation injury and tumor recurrence is often difficult because both conditions have similar clinical and radiologic presentations. This differentiation has been attempted using positron emission tomography (PET) with 18F-2-D-deoxyglucose (Di Chiro et al, 1985; 1987). However, in some series, low-grade gliomas and radiation injury were not consistently distinguishable. We have studied two patients presenting the clinical dilemma of radiationinduced encephalopathy versus recurrent tumor, using PET with the radiolabeled amino acid 11-D-L-methionine. Case \#1 developed clinical neurologic worsening and a fresh, contrast-enhancing CT lesion four months after completing radiotherapy for a thalamic astrocytoma (Grade 2). Case \#2 developed increasing and focal neurologic deficit, and a progressive, strongly enhancing $C T$ lesion at the site of the radiotherapy for her left frontal cystic astrocytoma. In case \#1, 11-C-Lmethionine (a) on an initial study showed no abnormal accumulation at the site of the CT abnormality, (b) following administration of the competing neutral amino acid L-phenylalanine, showed intense uptake of 11-C-L-methionine, in a distribution closely paralleling the CT abnormality. Case \#2 showed no detectible increase of 11-C-L-methionine accumulation at any point in the left frontal lobe, including the area of CT abnormality; she was not studied with L-phenylalanine. Case \#1 had biopsy confirmation of radiation necrosis; case \#2 had regression of clinical and radiologic abnormalities following steroid therapy: her course was considered typical of radiation necrosis. 
Our findings suggest that the mechanisms underlying accumulation of 11-C-L-methionine in areas of radiation necrosis are (a) non-concentrative (seen in both patients) and (b) diffusional (and therefore unaffected by pre-administration of L-phenylalanine) (seen in case \#1). Both mechanisms differ sharply from those encountered in brain tumor, where concentrative and saturable transport of $11-\mathrm{C}-\mathrm{L}-$-methionine has been noted (Bergstrom et al, 1987; O'Tuama et al, 1987). Hence this preliminary experience suggests that radiation necrosis and recurrent human brain tumor can be distinguished using two-phase 11-C-Lmethionine PET.

35.

\section{Neurotoxicity Study Following Intracarotid Administration of Cisplatin in Rats}

\section{S. SUGIMOTO, Y.L. YAMAMOTO, M. DIKSIC and W. FEINDEL (Montreal, Quebec)}

Although cisplatin chemotherapy has shown greater therapeutic effect against primary and metastatic malignant brain tumors when administered through the carotid artery than when given intravenously, toxicity to the brain following IC therapy has been a vexing problem. The pathophysiology of cisplatin toxicity is poorly understood. However, our evaluation of hemodynamic and metabolic aspects of the neurotoxicity following IC cisplatin in the rat may provide us with useful information for early detection of neurotoxicity.

Method - Female Wistar rats (190-250 g) divided into five groups underwent cannulation of the right carotid artery. Over 60 minutes they were given intracarotidly either $0.15-1.5 \mathrm{mg}$ of cisplatin or $1 \mathrm{ml}$ of normal saline (as a control). The $\mathrm{LD}_{10}$ of cisplatin with systemic administration in rats is known to be $1.6 \mathrm{mg} / \mathrm{kg}$. Blood-brain barrier (BBB) permeability, local cerebral blood flow (LCBF), local cerebral glucose utilization (LCGU), and local rate of protein synthesis using ${ }^{14} \mathrm{C}$ alpha aminoisobutyric acid (AIB), 18F-4-fluoroantipyrine (FAP), F-2-deoxy2-fluoro-2-D-glucose (FDG), and L-1-14C valine, respectively, were measured on Day I, 4 and 10 by quantitative double-tracer autoradiography. Studies of blood chemistry and histological evaluations were also made on Day 1, 4 and 10.

Results - When compared with the control group, the 0.15 $0.8 \mathrm{mg}$ group showed no hemodynamic or metabolic change. In the $1 \mathrm{mg}$ group, transient increase of BBB permeability on the ipsilateral side was noted on Day 1, but there was no apparent change on Day 4 or 10. Moderate reduction of LCGU on both sides, more obvious on the ipsilateral side, was noted on Day 4 and 10 . In the $1.2 \mathrm{mg}$ group, increase of BBB permeability on the ipsilateral side was noted on Day 1 and 4. Reduction of LCGU was noted even on Day 1 . There was the same tendency regarding the rate of protein synthesis as in the $1 \mathrm{mg}$ group. In the $1.5 \mathrm{mg}$ group, increased BBB permeability was noted in all cases on the ipsilateral side. Reduction of LCGU and the rate of protein synthesis was prominent on both sides on Day 1-10, and the difference between ipsi- and contra-lateral sides was more obvious in LCGU. No clear tendency was noted regarding LCBF in this study. Moderate and severe renal dysfunction were noted only in the $1.5 \mathrm{mg}$ group on Day 4 and 10. Structural damages in thalamus, hippocampus, caudate nucleus, globus pallidus, amygdala, hypothalamus, were noted only in the $1.5 \mathrm{mg}$ group $(60 \%)$.

Conclusion - Our multi-tracer autoradiographic studies indicate that a moderate reduction of LCGU associated with BBB permeability changes, as detected in the $1 \mathrm{mg}$ group on Day 4 and 10, are indicators of early neurotoxicity following IC cisplatin treatment.

36.

Superselective Intra-arterial Chemotherapy of Malignant Gliomas: Complications of Therapy with Special Emphasis on Central Neurotoxicity

J.F. EMRICH and J.-G. VILLEMURE (Montreal, Quebec)
Intra-arterial chemotherapy has been shown to be effective in increasing the concentration of drug delivered to high-grade gliomas. Clinical trials have shown the feasibility of this treatment. Preliminary indications are that it may increase survival in patients with high-grade gliomas. The possible deleterious effects of this method of treatment are related to the catheterization procedure itself and drug toxicity to the retina and central nervous system. Intra-arterial therapy has reduced or eliminated systemic drug toxicity and superselective infusion above the ophthalmic artery greatly reduces the risk of retinal toxicity. However, the higher concentrations of drug reaching the CNS can result in immediate and/or delayed cerebral toxicity which may affect the length or quality of survival by creating or aggravating a neurological deficit.

We analyzed the systemic, retinal and central neurotoxicity occurring in a group of 29 patients with recurrent high-grade gliomas treated with supra-ophthalmic, intra-arterial infusions of BCNU. We observed no systemic toxicity. Two patients $(7 \%)$ developed retinal toxicity after catheter displacement. Early cerebral toxicity was defined as a neurological deficit within $\mathbf{4 8}$ hours of treatment and not related to catheter induced vasospasm or thrombo-embolism. This occurred in 9 patients $(31 \%)$, was usually transient and was related to the concentration of BCNU infused. Late cerebral toxicity occurred in 4 patients $(14 \%)$ and manifested itself as a progressive decline in function without demonstrable tumor recurrence. The incidence and severity was related to the cumulative dose of BCNU. CT, MRI and PET scans were obtained in these patients and showed white matter atrophy and reduced glucose utilization in the treated hemisphere remote from the lumor.

Appreciation of the toxicities associated with this form of therapy is necessary to guide further refinements of technique, scheduling and dosing. Only then can intra-arterial chemotherapy be compared with intravenous administration.

37.

A New Approach to Steroid Therapy During Cranial Radiation (RT) for Brain Metastases (BM)

\section{D.E. WEISSMAN (Milwaukee, U.S.A.)}

The optimal dosing schedule of steroids to control brain edema from BM and limit steroid toxicity is unknown. "Standard therapy" includes dexamethasone (DEX) prescribed at a dose of $4 \mathrm{mg} \mathrm{q6h}$ and continued throughout the duration of RT and then slowly tapered over 1-3 weeks after RT is completed. This study was designed to test a b.i.d. schedule of DEX in patients (pts.) undergoing RT to answer:

1) Can b.i.d. dosing improve or stabilize deficits prior to starting RT?

2) Can steroids be safely tapered during RT and discontinued at the end of RT?

3) Will this regimen, having substantially less total steroid dose and duration of treatment, result in significantly less toxicity than "standard therapy"?

Pts. received $8 \mathrm{mg}$ b.i.d. $\times 4$ days then $4 \mathrm{mg}$ b.i.d. $\times 4$ days then $2 \mathrm{mg}$ b.i.d. until the final day of RT and then off. 13 pts. have been studied, 7 with a single $\mathrm{BM}$ and 6 with multiple $\mathrm{BM}$, with a mean performance score of 1.8 (ECOG). 10/13 pts. completed the prescribed steroid course; $1 \mathrm{pt}$. had DEX discontinued due to toxicity (steroid myopathy) but completed RT without neurologic progression, I pt. had a waxing and waning aphasia unresponsive to higher steroid doses and 1 pt. expired during treatment due to progressive visceral metastases. The mean duration of steroid treatment was $18 \pm 2$ days. 10/13 pts. received DEX for at least 24 hrs. prior to starting RT; 8 had neurologic improvement and 2 had stabilization at the time RT was begun. No pts. completing the prescribed course $(0 / 10)$ had neurologic progression within 30 days of stopping DEX. Five pts. developed steroid toxicity (two major, three minor).

Conclusions:

1) Twice daily dosing of DEX is an effective schedule for initial symptom control prior to starting RT.

2) Steroids can be safely tapered during RT and discontinued at the 
completion of RT.

3) Early data from this study suggests there is less steroid toxicity with this dosing regimen compared to standard therapy

38.

Steroids for Metastatic Cord Compression: Is High Dose More Effective Than Low Dose

\section{E. ARBIT, J.Y. DELATTRE, J.H. GALICICH and J.B. POSNER (New York, U.S.A.)}

Corticosteroids (CS) are an important part of the treatment of metastatic epidural cord compression, mainly because of their beneficial effect on vasogenic edema. Current treatment protocols call for the use of $96 \mathrm{mg}$ of dexamethasone per day, halving the dose each 2-3 days if the patient improves or stabilizes, and raising the dose if the patient experiences neurological deterioration. However, the risks of high dose $\mathrm{CS}$, in particular Gl perforation and infections, are well known. We have compared the effects of high dose versus low dose dexamethasone on neurological function in a non-neoplastic experimental spinal cord compression model in the rat. Seventeen Sprague-Dawley rats were studied. Once paraplegia occurred, the animals were randomized into three groups: untreated control $(n=5)$, high dose dexamethasone (HD, $1.25 \mathrm{mg} / \mathrm{kg}$ intramuscular b.i.d., $\mathrm{n}=5$ ), and low dose dexamethasone ( $\mathrm{LD}, 0.125 \mathrm{mg} / \mathrm{kg}$ intramuscular b.i.d., $\mathrm{n}=7$ ). Motor strength was evaluated daily by an observer unaware of the treatment given to the rats. Animals treated with dexamethasone (HD or LD) improved faster than untreated control. No significant difference in the rate of recovery or degree of motor improvement was noted between the HD and LD groups. Mortality was higher in the HD group due to infections or gastrointestinal perforation/bleeding.

\section{Conclusion}

Low dose dexamethasone may be as effective as high dose dexamethasone in protecting the spinal cord in face of epidural cord compression. These results have to be further validated prior to changing the ongoing protocols.

39.

Strategies in the Surgical Management of Epidural Spinal Metastases

\section{E. ARBIT and J.H. GALICICH (New York, U.S.A.)}

Although controversy still exists regarding the optimal treatment of patients with metastatic cord compression, there is a clear indication for surgery in patients (1) that have failed or are progressing on radiotherapy and (2) with structural abnormalities. The goals to be achieved by surgery are not mere decompression but rather reconstitution of the spinal canal and stabilization. For reconstitution of the canal, the approach must be tailored individually depending upon the location of the compressing mass. The anterior vertebral body resection and stabilization is used for anterior masses. A combined anterolateral approach is used for tumors that involve mainly the vertebral body and extend to the lateral or posterior elements. A posterolateral approach is used when tumors involve mainly the lateral elements and to some extent the lateral portion of the vertebral body. Laminectomy is reserved for cases of posterior compression. Anterior or posterior stabilization is invariably carried out in all cases except those that have minimal bony loss of the vertebral body or lateral elements either by neoplasm or surgery.
Dislocation, severe angulation, and motion segments involvement are best stabilized with posterior instrumentation.

Less than half of all patients that present with spinal cord compression reach surgery. In a series of 200 patients treated with vertebral body resection and stabilization $80 \%$ had neurological improvement, $80 \%$ had pain relief; morbidity was $10 \%$ and mortality $6 \%$. Out of 150 patients operated upon through posterior, posterolateral approaches, neurological improvement was present in $62 \%$ and pain alleviated in $50 \%$. Morbidity and mortality were similar in both groups. The median survival for anterior operations was 12 months, and for posterior only 7 months. The reason for the different outcome in the two patient populations is the more frequent performance of posterior operations for the purpose of tissue diagnosis, pain relief, and neurologic salvage.

The following papers were presented at the 3rd Canadian NeuroOncology Meeting and the abstracts published in the Can J Neurol Sci 1988; 15(2)

Flanking Markers Define the Limits of the Bilateral Acoustic Neurofibromatosis Locus. G.A. ROULEAU, B.R. SEIZINGER, W. WERTELECKI, J.L. HAINES, D.W. SUPERNEAU and J.F. GUSELLA (Boston, U.S.A.) Abst. \#53

The Effect of Ibuprofen Therapy on Patients with Peritumoral Edema. R.F. DEL MAESTRO and A.G. MATTAR (London, Ontario) Abst. \#174

Phosphorus Magnetic Resonance Spectroscopy and Characterization of Astroctyomas, Meningiomas and Pituitary Adenomas. J.F. EMRICH, E.A. SHOUBRIDGE, J.G. VILLEMURE, W. FEINDEL and D.L. ARNOLD (Montreal, Quebec) Abst. \#169

Analysis of Human Cerebral Tumors by Nuclear Magnetic Resonance Spectroscopy, Microgravimetry and Histopathology. J.F. MEGYESI and R.F. DEL MAESTRO (London, Ontario) Abst. \#167

${ }_{1 \mathrm{H}}$ and ${ }_{13 \mathrm{C}}$ Nuclear Magnetic Resonance (NMR) Spectroscopy of Plasma from Patients with Primary Intracranial Neoplasms. G.R. SUTHERLAND, J. PEELING and K. MARAT (Winnipeg, Manitoba) Abst. \#168

Correlation of CT and MRI with Physiological Monitoring and Histopathology in Stereotatic Biopsies. J. GORECKI, E.J. DOLAN, R.R. TASKER and W. KUCHARCZYK (Toronto, Ontario) Abst. \#166

Platelet Accumulation in Cerebral Mass Lesions. R.F. DEL MAESTRO, D.R. GRAVELLE, G.J. MORRISSEY, C.L. FARRELL and A.A. DRIEDGER (London, Ontario) Abst. \#176

Carcinomatous Meningitis After Intracranial Surgery: A Retrospective Study. J.F. MEGYESI and R.F. DEL MAESTRO (London, Ontario) Abst. \#182

The Effect of Ibuprofen on Tumor Growth and Vascular Permeability in a Rat Glioma Model. C.L. FARRELL, J.F. MEGYESI and R.F. DEL MAESTRO (London, Ontario) Abst. \#175

Changes of Blood Brain Barrier Permeability and Local Cerebral Blood Flow Following Intracarotid Administration of BCNU in Rats. S. NAGAHIRO, Y.L. YAMAMOTO, M. DIKSIC and W. FEINDEL (Montreal, Quebec) Abst. \#173

Anterior Decompression and Fixation for Symptomatic Spinal Metastases. R.G. PERRIN and R.J. McBROOM (Toronto, Ontario) Abst. \#180 